Revue scientifique sur la conception et l'aménagement de l'espace

$5 \mid 2011$

Le jardin et ses rapports à l'art. Encadrer, décadrer, recadrer

\title{
Phénoménologie du paysage
}

Phenomenological approach of landscape

\section{Françoise Dastur}

\section{(2) OpenEdition}

1 Journals

\section{Édition électronique}

URL : https://journals.openedition.org/paysage/21559

DOI : 10.4000/paysage.21559

ISSN : 1969-6124

\section{Éditeur :}

École nationale supérieure du paysage de Versailles-Marseille, Institut national des sciences appliquées Centre Val de Loire - École de la nature et du paysage, École nationale supérieure d'architecture et de paysage de Bordeaux, École nationale supérieure d'architecture et de paysage de Lille, Agrocampus Angers

\section{Référence électronique}

Françoise Dastur, «Phénoménologie du paysage », Projets de paysage [En ligne], 5 | 2011, mis en ligne le 20 janvier 2011, consulté le 16 octobre 2021. URL : http://journals.openedition.org/paysage/21559 DOI : https://doi.org/10.4000/paysage.21559

Ce document a été généré automatiquement le 16 octobre 2021.

\section{c) (7) $\Theta$}

La revue Projets de paysage est mise à disposition selon les termes de la Licence Creative Commons Attribution - Pas d'Utilisation Commerciale - Pas de Modification 4.0 International. 


\title{
Phénoménologie du paysage
}

\author{
Phenomenological approach of landscape
}

\section{Françoise Dastur}

1 Le terme de paysage, construit sur pays, semble d'abord ne pouvoir renvoyer qu'à l'espace rural, à la campagne, au point que l'on pourrait se demander si l'idée même de "paysage urbain», expression pourtant utilisée aujourd'hui, n'est pas absurde. Pourtant il n'y a pas de paysage « en soi », qui existerait en dehors de l'œil et du travail humains, puisque le terme même de "pays », issu du latin pagus, qui signifie village ou canton, désigne par lui-même un espace délimité et organisé par l'homme, comme le dit le verbe pango, auquel se rattache pagus, et qui a le sens de planter ou d'enfoncer des piquets, et par là de fixer les limites d'une portion de terre bien définie. Certes le terme de paysage, dans son usage actuel, désigne ce que l'on voit selon une large perspective déployant à l'arrière des objets les plus proches un horizon qui peut apparaître comme indéfiniment éloigné. C'est cette idée du paysage que nous a transmise la peinture moderne, tout particulièrement la peinture romantique, anglaise et allemande, avec la représentation d'une nature sublime et incommensurable (qu'on pense par exemple à Caspar David Friedrich et à son célèbre tableau intitulé Un voyageur contemplant une mer de nuages), mais aussi sauvage et déchaînée (dans les peintures marines de Turner).

2 Si le terme de paysage a donc d'abord désigné le "pays ", c'est-à-dire l'ensemble des formes et des reliefs d'un espace limité, il implique pourtant nécessairement un point de vue et la présence d'un observateur devant lequel l'horizon se déploie. Le paysage est en effet essentiellement le résultat d'une expérience visuelle, comme le montre bien le terme qu'on lui substitue souvent de "panorama», mot inventé en 1792 par le peintre Robert Barker à partir du grec pan (tout) et orama (vue) pour désigner les peintures qu'il effectuait sur une surface cylindrique, première technique permettant une vue en grand angle. Il semble cependant que, pour qu'il y ait vraiment paysage, il faille demeurer à une distance qui demeure celle de la vision normale de l'œil humain, car une vue prise d'avion par exemple est la représentation d'un espace totalement objectivé où nul relief ne peut plus être perçu. Non seulement donc le paysage exige la présence de l'œil humain, mais aussi celle de la main humaine, puisque c'est par le travail de celui qui habite le pays, le paysan, que l'espace terrestre a été, en partie au 
moins, configuré. Qu'on songe ici non seulement aux changements apportés par le travail du sol, par la construction des voies de transport, mais aussi par ceux résultant de l'implantation de nouvelles espèces importées d'ailleurs dans un territoire donné. Le paysage, même lorsqu'il résulte en grande partie de l'action humaine et du travail des paysans, n'est pourtant pas pour ces derniers un objet d'expérience, puisqu'ils ne perçoivent leur entourage que d'un point de vue utilitaire et dans la perspective d'une rentabilité possible. Il faut donc, pour que le paysage apparaisse, et que la nature se montre dans ce qu'elle a d'inhumain et de non dompté, que le regard cesse d'être « intéressé » et qu'il s'ouvre à ce que l'on pourrait nommer la " pure » apparition de ce qui est.

3 C'est de ce regard " désintéressé » dont il est question dans la phénoménologie. Un tel regard est en effet le résultat de la méthode phénoménologique, celle qui permet l'accès aux phénomènes, à ce qui se manifeste de soi-même, et qui repose sur cette opération que Husserl nomme epokhè, reprenant ainsi un terme issu du scepticisme ancien qui signifie la mise « entre parenthèses » ou la mise « hors circuit » de toutes les préconceptions, à la fois pratiques et théoriques, qui guident habituellement le regard que nous portons sur les choses. Ainsi pour que le paysage apparaisse, il faut que le paysan laisse tomber ses outils, mais il faut aussi que le géographe et le botaniste cessent de percevoir dans le territoire et les plantes qui le couvrent des "objets" d'investigation et de recherche. Heidegger a ainsi mis l'accent sur le fait qu'au niveau de la quotidienneté, toutes choses, y compris les choses dites "naturelles» nous apparaissent sous l'angle de leur utilité, de sorte que nous ne les voyons pas en « ellesmêmes ", et qu'il en va de même dans la perspective scientifique, où elles sont réduites à un ensemble de variables quantitatives. Il s'agit donc, si nous voulons comprendre ce qu'est un paysage, que nous revenions à cette expérience que Merleau-Ponty a nommée " primordiale » et qui est celle d'une perception qui n'a pas encore fait du monde et des choses de purs « objets " pour un sujet qui vise à se les approprier de manière pratique ou théorique. Une telle expérience "primordiale » du monde est en réalité déjà par elle-même celle dans laquelle s'installe l'artiste, et tout particulièrement le peintre, lorsqu'il entreprend de « laisser » les choses apparaître d'elles-mêmes à son regard afin d'être alors en mesure de les représenter. Ce qui d'une certaine manière précède l'art et le rend possible, c'est donc cette expérience spirituelle qu'a si bien décrite le peintre chinois et moine bouddhiste du XVII ${ }^{e}$ siècle Shitao dans Les Propos sur la peinture du moine Citrouille-Amère. Il s'agit en effet pour le peintre, explique-t-il dans ce traité fondamental pour toute la peinture chinoise ultérieure, de parvenir d'abord, à force de contemplation, à faire un avec le sujet contemplé, afin de pouvoir ensuite exécuter son tableau par touches rapides et avec une grande économie de traits. L'idée fondamentale en est celle de «l'unique trait de pinceau » qui n'autorise ni retouches ni pauses dans l'exécution et qui incarne le trait d'union entre l'homme et l'univers et la conjonction intime, de la nature intérieure et de la nature extérieure.

4 Le peintre n'apporte pas seulement son œil, mais tout son corps, comme le souligne avec pertinence Merleau-Ponty dans le petit essai entièrement consacré à la peinture qu'est L'Eil et l'Esprit. Car pour que le paysage apparaisse à nos yeux, il faut en effet, cela a déjà été souligné, une sorte de vision qui ne soit pas objectivante et qui naisse pour ainsi dire du milieu du paysage lui-même. Le paysage correspond en effet à une perception horizontale, celle qu'a l'œil humain de son environnement, un environnement dont l'observateur fait lui-même partie. Il n'y a en effet d'horizon, comme le mot l'indique bien, qui vient du grec horos, qui signifie limite, qu'en rapport 
avec ce centre de perception qu'est le corps humain, dont Husserl disait dans le second volume de ses Idées directrices, qu'il est le " point zéro » à partir duquel l'espace peut se déployer, et un «ici » absolu, à partir duquel il peut y avoir un là et un là-bas. Certes ce point zéro qu'est mon corps propre, c'est-à-dire mon corps vécu « de l'intérieur », peut se mouvoir et par là changer son système d'orientation, mais de son propre point de vue, il demeure immuable : je ne peux en effet m'éloigner de mon corps, qui est ainsi une chose tout à fait particulière de mon monde. Ma perception en est limitée : je ne peux voir ni mon dos ni mon visage. Il n'y a donc pas d'observateur absolu, devant lequel s'étalerait un espace pur, dépourvu de profondeur et de zones cachées.

5 Cette analyse husserlienne du rapport du sujet incarné à l'espace a été prolongée par Heidegger qui, donnant à l'être de l'homme le nom de Dasein, mot qui signifie littéralement être là, insiste sur le fait que cet existant qu'est l'homme a un rapport essentiel à l'espace, ce qui implique que son rapport à l'espace ne s'ajoute pas du dehors à la détermination de son être. Mais ce rapport à l'espace qui le constitue ne peut pas être compris comme un rapport à ce milieu dans lequel se tiennent les corps dont nous parle la philosophie de l'âge classique. Car les analyses de Heidegger se situent elles aussi au niveau de l'expérience quotidienne et à l'intérieur de ce que Husserl nommait le «monde de la vie ». À ce niveau, le rapport de l'homme à l'espace ne passe pas par la mesure objective de la distance qui sépare deux corps, mais il se joue sur un plan beaucoup plus fondamental : pour pouvoir en effet mesurer une distance, il faut que l'éloignement du corps par rapport auquel je me situe m'ait déjà été révélé. Or ce qui me révèle l'éloignement, ce n'est pas l'attitude théorique qui est celle que j'adopte lorsque j'évalue objectivement une distance, mais une attitude pratique que Heidegger nomme Besorgen, préoccupation. La préoccupation, explique Heidegger, se manifeste par une tendance générale au rapprochement de l'objet dont elle se préoccupe et c'est d'ailleurs cette tendance au rapprochement qui est à l'origine de bien des inventions de la technique moderne, de la radio au téléphone et à l'avion. L'espace ne se déploie donc originairement pour le Dasein qu'au travers de son rapport pratique au monde. Certes l'être humain occupe une place dans l'espace, du fait qu'il est spatial en son essence, mais ce n'est pas un hasard si Heidegger dit de lui qu'il est là et non pas simplement ici. Car contrairement à une chose corporelle quelconque, à laquelle on peut assigner un lieu précis dans l'espace, l'être humain n'est véritablement que « là » où se nouent pour lui tous les rapports aux choses et aux autres êtres vivants qui constituent son monde.

Ce qu'indique donc le mot même de paysage, c'est le rapport qu'entretient l'être humain avec l'espace ; un rapport à la fois corporel et existentiel. C'est en effet par son corps que l'être humain est dans l'espace, ou plutôt, comme le souligne avec pertinence Merleau-Ponty, qu'il « habite » l'espace et qu'il a donc avec lui un rapport si intime que l'on peut dire qu'il n'y aurait pas d'espace du tout pour lui s'il n'avait pas de corps. C'est de cet espace habité et donc toujours déjà orienté, au sens où il a un haut et un bas, une gauche et une droite, que l'on fait l'expérience quand on regarde un paysage, lequel n'est pas tant d'ailleurs "devant» nous qu' «autour » de nous. C'est ce qui explique que nous ayons, avec les choses qui sont éloignées de nous dans l'espace, un rapport qui ne peut être mesuré de manière objective, car cette troisième dimension qu'est la profondeur est la plus « existentielle» de toutes les dimensions de l'espace. On sait que la représentation de la perspective a été, à la Renaissance, l'objet d'études et de codifications savantes. Mais cette reconstitution d'un espace objectif, selon les présupposés d'une vision monoculaire et d'un spectateur immobile, ne restitue en rien l'expérience concrète qui est la nôtre, lorsque nous balayons des yeux l'ensemble des 
éléments d'un paysage et que nous changeons constamment de positions pour en obtenir une vision aussi globale que possible. La profondeur est en effet, comme Merleau-Ponty l'a bien montré, une dimension qui implique en elle-même le regard humain, et elle ne peut jamais être considérée comme une dimension objective du fait qu'elle n'est pas seulement une dimension spatiale, mais aussi une dimension temporelle, puisque la distance qui sépare le spectateur d'une chose éloignée peut aussi être mesurée en unités de temps. Ce dont nous faisons donc l'expérience lorsque nous regardons un paysage, c'est moins d'une portion d'espace que d'un moment du monde, qui ne peut se révéler à nous que parce que, par les mouvements de notre corps, nous appartenons à la même spatio-temporalité qui le constitue. Il semble donc que la représentation de ce qu'est notre perception concrète du paysage ne puisse être l'objet ni de cette technique du dessin qu'est la perspective, ni même de cette technique moderne qu'est la photographie, qui présuppose elle aussi l'immobilité de l'œil photographiant. Il n'y aurait ainsi d'«art " photographique que celui qui tendrait à restituer la vision concrète, c'est-à-dire l'expérience vécue de la profondeur et le bougé de la perception.

7 Le rapport que nous entretenons avec l'espace, parce qu'il est essentiellement corporel, est aussi et d'abord existentiel. C'est ce qui explique la phrase devenue célèbre « Le paysage est un état d'âme " que l'on trouve dans le Journal d'Amiel, écrivain et philosophe suisse, qui suivit à Berlin dans les années 1840 l'enseignement de ce philosophe par excellence de la nature que fut Schelling.

8 S'agit-il là d'une simple forme d'anthropomorphisme ? Ce le serait en effet si l'espace et la nature avaient une existence « en soi » sur laquelle l'être humain pourrait projeter sa propre manière de sentir. Mais la nature, tout comme l'espace, n'existe pour nous que dans ce qu'elle nous donne à voir, ce qui ne veut certes pas dire qu'elle serait une création humaine, mais que ce qu'elle est en elle-même "aime à se cacher » comme le disait déjà Héraclite, et ne livre pas à notre regard. C'est ce qui explique que, comme Ludwig Binswanger, psychiatre suisse d'obédience phénoménologique, l'a bien montré, il y a une profonde relation entre l'espace vécu et la situation affective et corporelle du sujet. Cette relation apparaît clairement dans certaines pathologies, telles que la schizophrénie, dans laquelle l'espace perd précisément sa fonction d'entourage et apparaît comme un lieu dénué de sens et irréel, alors que dans la manie, le rapport affectif à l'espace, de terrifiant qu'il est pour le schizophrène, se teinte d'indifférence, de sorte que le maniaque, qui ne vit que dans le présent, n'a plus aucun sens de ce que serait un chez-soi. L'espace affectif et existentiel du paysage précède donc l'espace objectif et mesurable, comme le montre d'ailleurs l'exemple de ces hommes que l'on nomme " primitifs » qui n'ont nul besoin de repères pour s'orienter, précisément parce que les directions et les positions spatiales ont uniquement pour eux une signification affective. C'est une telle expérience du paysage que les peintres modernes, de Cézanne aux impressionnistes, ont tenté d'exprimer dans leurs tableaux.

9 Car le paysage, s'il ne se réduit pas à un morceau d'espace, mais implique aussi les dimensions temporelles, corporelles et affectives, ne peut être défini à partir de la seule expérience visuelle. Certes, notre rapport au monde n'est rendu possible que par le biais de nos cinq sens, et chacun d'eux ne nous en révèle qu'un aspect. Pourtant, comme Merleau-Ponty le souligne avec raison, par-delà leur absolue incommunicabilité, nos sens sont structurellement ouverts les uns aux autres, de sorte que notre expérience du paysage n'est jamais uniquement visuelle, mais qu'elle 
requiert également le concours de nos autres sens : de notre sens auditif (que serait en effet la perception de la mer sans le bruit des vagues, ou celle d'une forêt sans celui du vent dans les arbres?), de notre sens olfactif (le parfum du thym sauvage dans la garrigue), voire de notre sens gustatif (le goût de sel apporté par la proximité de la mer) et de notre sens tactile (le velouté du feuillage et la rugosité de la roche). C'est donc cette expérience, dans son entièreté, qu'il s'agit pour le peintre moderne de rendre sur la toile. On reconnaît là ce qui a conduit les impressionnistes à donner tant d'importance à la pure sensation. Il semble bien en effet que les impressionnistes, qui s'appuyaient sur les travaux que le physicien Eugène Chevreul avait consacrés à la décomposition de la lumière, ont ainsi été conduits à privilégier la seule sensation visuelle. C'est donc sur une théorie scientifique, plutôt que sur leur propre expérience perceptive, qu'ils ont fondé leur peinture. Or, l'expérience perceptive, comme l'a bien montré Merleau-Ponty, ne consiste nullement en l'imposition d'une forme sur la donnée préalable d'une mosaïque de sensations, mais bien en la saisie immédiate d'une figure qui se dessine de façon mouvante sur un fond. Ce qui se donne donc d'emblée, c'est cette configuration dynamique qu'est le paysage et non pas des données sensorielles dont la multiplicité devrait être synthétisée par un acte ultérieur de l'intellect. Il faut d'ailleurs souligner que, pour parvenir à rendre fidèlement sur la toile les «impressions » que Monet considérait comme les «données immédiates » de la perception, il lui a fallu non point partir de la nature sauvage, mais bien du jardin qu'il mit tant de soin à organiser chez lui, à Giverny. Ses tableaux, tout particulièrement les derniers, sont donc ceux d'une nature apprivoisée et devenue un pur décor. Or, la véritable expérience du paysage n'est jamais celle d'un décor, mais au contraire de la configuration mouvante d'un espace dans lequel on chemine et qui ne prend précisément forme que par ce cheminement. C'est cette configuration naissante du paysage, au moment où un premier regard humain commence à se poser sur lui, qu'a tenté de peindre Cézanne dans ses tableaux représentant la montagne Sainte-Victoire.

\section{BIBLIOGRAPHIE}

Binswanger, L., Le Problème de l'espace en psychopathologie, traduit et préfacé par C. Gros, Toulouse, Presses universitaires du Mirail, 1998.

Husserl, E., Idées directrices pour une phénoménologie, Paris, Gallimard 1950.

Husserl, E., Recherches phénoménologiques pour la constitution, Paris, PUF, 1982.

Heidegger, M., Etre et Temps, Paris, Authentica, 1985 ; Paris, Gallimard, 1986.

Merleau-Ponty, M., L'CEil et l'Esprit, Paris, Gallimard, 1964.

Shitao, Les Propos sur la peinture du moine Citrouille-amère, traduction et commentaire de P.

Ryckmans, Paris, Hermann, 1984.

Projets de paysage, 5 | 2011 


\section{RÉSUMÉS}

On se propose de montrer ici que la notion même de paysage ne peut être comprise que de manière phénoménologique, dans la mesure où seul un regard désintéressé et dénué de toute visée pratique ou théorique peut nous ouvrir à la pure apparition de ce qui est. C'est dans une telle attitude par rapport à la nature que s'installe le peintre, lequel vise avant tout à laisser les choses apparaître d'elles-mêmes. Le rapport qu'entretient l'être humain avec l'espace se révèle être alors primordialement un rapport existentiel et affectif. Cela implique aussi que le paysage ne puisse plus être mis en relation avec une expérience purement visuelle, mais qu'il requiert également le concours des autres sens. C'est cette expérience d'un rapport à la fois corporel et configuratif au paysage que la peinture moderne s'est donnée pour tâche de restituer.

The purpose of this paper is to show that the notion of landscape can be understood only in a phenomenological manner, since a disinterested look devoid of any practical or theoretical intention alone can open us to the pure appearance of what is. This is what defines the painter's attitude in regard to nature, in so far as he wants to let the things appear from themselves. The human being's relation to space reveals therefore itself to be of an existential and affective nature. But this implies that the landscape can no longer be connected to a mere visual experience, but that it requires also the mediation of the other senses. The restitution of this at the same time corporeal and configurative experience of landscape has become a task for modern painting.

\section{INDEX}

Keywords : phenomenology, landscape, space, painting, body

Mots-clés : phénoménologie, paysage, espace, peinture, corps

\section{AUTEUR}

\section{FRANÇOISE DASTUR}

Françoise Dastur est professeur honoraire de philosophie, rattachée aux Archives Husserl de Paris (ENS ULM), a enseigné dans les universités de Paris 1, Paris 12 et Nice-Sophia Antipolis. Elle est présidente honoraire de l'École française de Daseinsanalyse dont elle fut l'un des membres fondateurs.

dastur[at]club-internet[dot]fr 